\title{
Basic Study of Radial Distributions of Electromagnetic Vibration and Noise in Three-Phase Squirrel-Cage Induction Motor under Load Conditions
}

\author{
Isao Hirotsuka*, Kazuo Tsuboi* and Yousuke Takahashi**
}

\begin{abstract}
Reduction of electromagnetic vibration and acoustic noise from three-phase squirrel-cage induction motors (IMs) is very important, particularly from the standpoint of environmental considerations. Although the electromagnetic vibration of IMs has been studied for several years, the relationships between the radial distribution of the electromagnetic vibration and noise and the electromagnetic forces responsible for them have not yet been analyzed in sufficient detail. In the present study, we investigated this relationship experimentally for a small IM under different load conditions. Our results clearly show that the radial distributions of the dominant electromagnetic vibration and noise components match the mode shape of the dominant electromagnetic force producing these components.
\end{abstract}

Keywords: Induction motor, Noise, Vibration, Harmonic flux.

\section{Introduction}

In recent years, the demand for induction motors (IMs) with low noise characteristics has increased considerably owing to environmental considerations. The basic concepts of IMs have been clarified through numerous studies [1]. However, the relationships between the radial distributions of the electromagnetic vibration and noise and the force waves responsible for them have not yet been analyzed in sufficient detail.

In this paper, we present the results of an experimental investigation into the radial distributions of the electromagnetic vibration and noise generated by an IM under various load conditions. First, we list the occurrence frequency and mode of the dominant electromagnetic force generated by the interaction of the harmonic fluxes in the IM gap. Next, we describe the experimental methods. Finally, on the basis of the results from several trials, we show that the radial distributions of the dominant electromagnetic vibration and noise components clearly correspond to the mode shape of the dominant electromagnetic forces.

\section{Dominant Electromagnetic Forces}

The general equations for the dominant electromagnetic

\footnotetext{
* Department of Electrical Engineering, Chubu University, Japan

** Toshiba Industrial Products Mfg., Japan

Received 08 April 2013 ; Accepted 30 April 2013
}

forces responsible for the dominant electromagnetic vibration and noise components are derived in [2]. Table 1 summarizes the occurrence frequency $f_{M}(\mathrm{~Hz})$ and mode $M$ of the dominant electromagnetic force in an IM driven by a sinusoidal power supply, where ns and $\mathrm{n}$ denote the numbers of stator and rotor slots, respectively; $p^{\prime}$ denotes the number of pole pairs; s denotes the slip; $f$ denotes the frequency of the power supply; and $L$ and $K$ are integers indicating the orders of the stator and rotor slot permeances, respectively. For convenience, the dominant forces are allocated a classification number. This table also shows that two types of electromagnetic forces [I] and [II] are produced under different load conditions. Force [I] is generated by the interaction between the stator fundamental flux and the rotor harmonic flux under both no load and load conditions, whereas force [II] is generated by the interaction between the stator harmonic flux and the rotor fundamental flux only under load.

Table 1. Occurrence frequency and mode of dominant

\begin{tabular}{|c|c|c|}
\hline $\begin{array}{c}\begin{array}{c}\text { Classification } \\
\text { number }\end{array} \\
\end{array}$ & Frequency $f_{v}$ & Mode $M$ \\
\hline (1) & $\left|(1-s) K n / p^{\prime}-2\right| f$ & $\mid \begin{array}{ll}\text { [I] } & \left|\left(p^{\prime}\right)+\left(p^{\prime}+L n_{s}-K n\right)\right| \\
{[\text { [II }]} & \left|\left(p^{\prime}+L n_{s}-K n\right)+\left(p^{\prime}\right)\right|\end{array}$ \\
\hline (2) & $\left|(1-s) K n / p^{\prime}\right| f$ & {$\left[\begin{array}{l}\text { [I] }\left|\left(p^{\prime}\right)-\left(p^{\prime} \pm L n_{s} \mp K n\right)\right| \\
{\left[\text { [II] }\left|\left(p^{\prime} \pm L n_{s} \mp K n\right)-\left(p^{\prime}\right)\right|\right.}\end{array}\right.$} \\
\hline (3) & $\left|(1-s) K n / p^{\prime}+2\right| f$ & {$\left[\begin{array}{ll}\text { II] } & \left|\left(p^{\prime}\right)+\left(p^{\prime}-L n_{s}+K n\right)\right| \\
{[\text { [II] }} & \left|\left(p^{\prime}-L n_{s}+K n\right)+\left(p^{\prime}\right)\right|\end{array}\right.$} \\
\hline
\end{tabular}




\section{Test Motor and Measurements}

A motor (specifications listed in Table 2) with 36 and 33 stator and rotor slots, respectively, was used as the test motor; this slot combination is not used commercially but simplifies examination of the dominant electromagnetic vibration and noise. A sinusoidal voltage of variable frequency was supplied, and the natural frequencies of the test motor were measured by using an impact hammer at the same motor temperature as that for the measurements of the electromagnetic vibration and noise. It was confirmed that the natural frequencies of the test motor were approximately constant even if the rotors were replaced.

Noise measurements were conducted in a motor noise laboratory in which reflected sound and background noise were minimized (Fig. 2). The test motor was set on an eddy current dynamometer with a damper rubber to minimize the effects of external vibrations. To avoid abnormal starting phenomena in the test motor and to adjust the supplied frequency f, a sine wave variable frequency power supply was used. The radial vibration was detected by two accelerometers: one fixed on top of the IM, and the other, movable. The signals from these accelerometers were input into a fast Fourier transform (FFT) analyzer through charge amplifiers. Electromagnetic noise was also detected by two devices: a fixed microphone mounted at the upper center position on the IM stator and a movable microphone. The measured sound pressure levels $(\mathrm{dB})$ were analyzed by the FFT analyzer via sound level meters. Finally, to estimate the electromagnetic noise in better detail, the distance between the center of the test motor and both microphones was $0.5 \mathrm{~m}$, which is less than the stipulated distance in JIS C 4210 [3].

Table 2 .Specifications of test motor

\begin{tabular}{c|c}
\hline Number of poles: $2 p^{\prime}$ & 4 \\
\hline Rated output & $1.5 \mathrm{~kW}$ \\
\hline Rated voltage & $200 \mathrm{~V}$ \\
\hline Rated frequency & $60 \mathrm{~Hz}$ \\
\hline Number of stator slots $n_{s}$ & 36 \\
\hline Number of rotor slots $n$ & 33 \\
\hline Skew of rotor slot & No skew \\
\hline Main natural frequencies & $506,1043,1344,1684$, \\
& $1956,2653,3313,3806 \mathrm{~Hz}$ \\
\hline
\end{tabular}

Measurements of vibration and noise were performed at no load (i.e., when the percentage of the load to the rated load, $\mathrm{Lp}$ is $0 \%$ ) and rated load ( $\mathrm{Lp}=100 \%)$. When $\mathrm{Lp}$ is changed, the occurrence frequency of the vibration and noise component, $\mathrm{fM}$ is varies because of the change in slip s. To eliminate the effects of the mechanical system and keep fv constant, the voltage/frequency ratio V/f was kept at 200/60 while adjusting either V or f. Further, when force
(2) in Table I is the cause of vibration and noise component, $\mathrm{fM}$ can be maintained at constant value by adjusting V/f and keeping the rotational speed at 1800 min-1 to avoid the effect of the natural frequency.

\section{Experimental Results}

\subsection{Dominant Electromagnetic Vibration and Noise Components}

As shown in Fig. 1, fv decreases with an increase in the load because of the accompanying increase in slip. Moreover, the figure clearly shows that the dominant electromagnetic noise component has the same frequency as the dominant electromagnetic vibration component. In this paper, the five components listed in Table III are considered to describe the radial distributions, which are discussed in the next subsection.

As summarized in Table III, the dominant components correspond to the forces with small modes M (Table I). In particular, $1109 \mathrm{~Hz}$ and $2100 \mathrm{~Hz}$ are dominant at $\mathrm{Lp}=0 \%$ because they correspond to forces with $\mathrm{M}=1$ and 2 , respectively.

\subsection{Radial Distribution of Dominant Electromagnetic Vibration and Noise Components}

Fig. 4 shows the radial distributions of the $991 \mathrm{~Hz}$ (= $16.5 \mathrm{f})$ vibration and its noise components at any instant at $\mathrm{Lp}=0 \%$. These distributions are similar to each other and correspond to $\mathrm{M}=3$, as shown in Table III. Fig. 5 shows the corresponding distributions at $\mathrm{Lp}=100 \%$. In this figure, the rotational direction of the node is also indicated. These distributions also appear to correspond to $\mathrm{M}=3$.

Thus, from Figs. 4 and 5, it is observed that the distribution of the electromagnetic vibration and noise component do not change form regardless of the load. Figs. $6,7,8$, and 9 show the radial distributions of the 1109 (18.5f), 1978 (33f), 2100 (35f), and $3088 \mathrm{~Hz}$ (51.5f) vibration and noise components at any instant. This control can regulate capacitor voltages to the reference value per arm module

Table 3. Dominant electromagnetic vibration and noise components

\begin{tabular}{c|c|c|c}
\hline$f_{\mathrm{v}}$ at $L_{\mathrm{p}}=0 \%$ & $M$ & $K$ & $L$ \\
\hline $990(16.5 f)$ & 3 & 1 & 1 \\
\hline $1109(18.5 f)$ & 1 & 1 & 1 \\
\hline $1978(33 f)$ & 6 & 2 & 2 \\
\hline $2100(35 f)$ & 2 & 2 & 2 \\
\hline $3088(51.5 f)$ & 5 & 3 & 3 \\
\hline
\end{tabular}




\section{Conclusions}

We investigated the radial distribution of the vibration and noise of an IM under different load conditions. Our experiments confirmed that a close relationship exists between the radial distributions of the electromagnetic
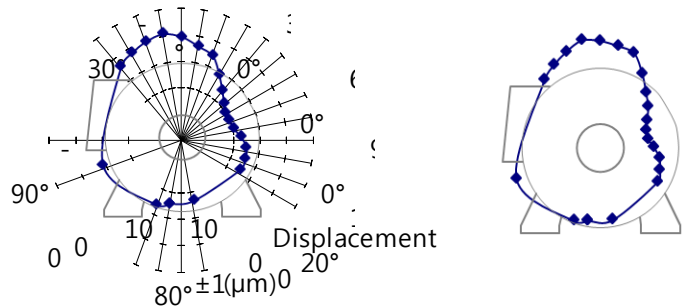

(1) at base time

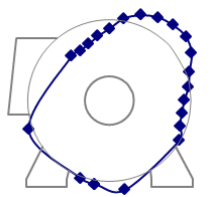

(5) after $1 / 991 \times 4 / 12(\mathrm{~s})$

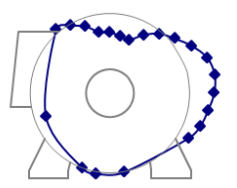

(9) after $1 / 991 \times 8 / 12(\mathrm{~s})$

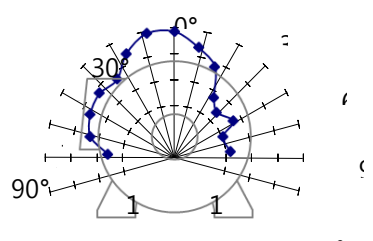

Sound pressure level $\left(\mathrm{kg} / \mathrm{m}^{2}\right)$ (1) at base time

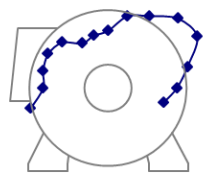

(5) after $1 / 991 \times 4 / 12(\mathrm{~s})$

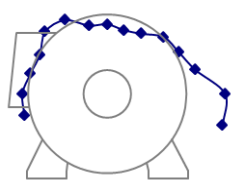

(9) after $1 / 991 \times 8 / 12(\mathrm{~s})$
(2) after $1 / 991 \times 1 / 12(\mathrm{~s})$

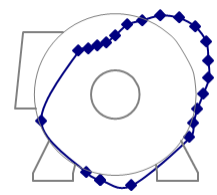

(6) after $1 / 991 \times 5 / 12(\mathrm{~s})$

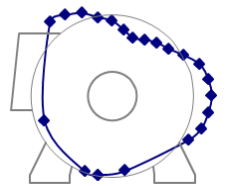

(10) after $1 / 991 \times 9 / 12(\mathrm{~s})$
(2) after $1 / 991 \times 1 / 12(\mathrm{~s})$

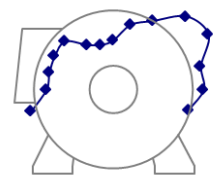

(6) after $1 / 991 \times 5 / 12(s)$

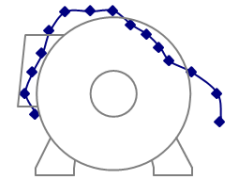

(10) after $1 / 991 \times 9 / 12(\mathrm{~s})$

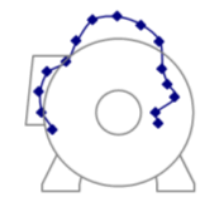

vibration and noise components and the radial forces. We believe that the results of our study can assist in noise and vibration reduction for IMs. And the control can regulate capacitor voltages to the reference value per arm module.

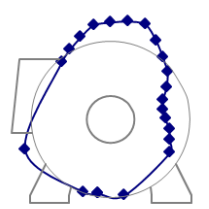

(3) after $1 / 991 \times 2 / 12(s)$

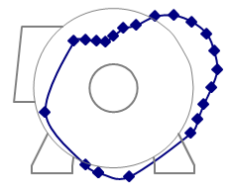

(7) after $1 / 991 \times 6 / 12(s)$

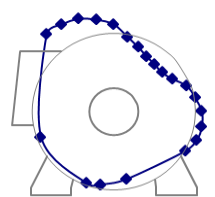

(11) after $1 / 991 \times 10 / 12(s)$

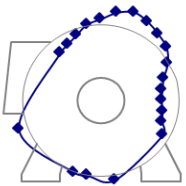

(4) after $1 / 991 \times 3 / 12(s)$

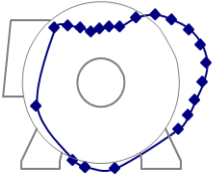

(8) after $1 / 991 \times 7 / 12(s)$

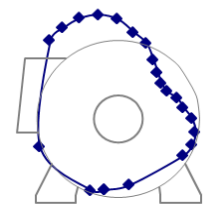

(12) after $1 / 991 \times 11 / 12(\mathrm{~s})$

(a) Electromagnetic vibration.

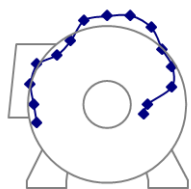

(3) after $1 / 991 \times 2 / 12(\mathrm{~s})$
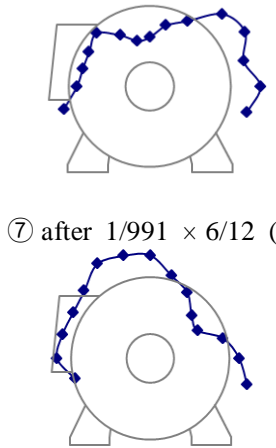

(11) after $1 / 991 \times 10 / 12(\mathrm{~s})$

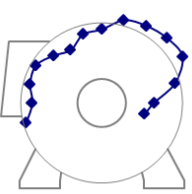

(4) after $1 / 991 \times 3 / 12(s)$

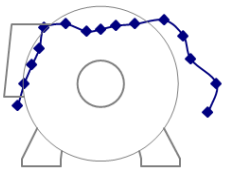

(8) after $1 / 991 \times 7 / 12$ (s)

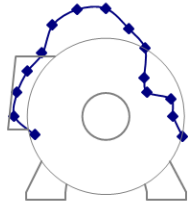

(12) after $1 / 991 \times 11 / 12(\mathrm{~s})$

(b) Electromagnetic noise. 


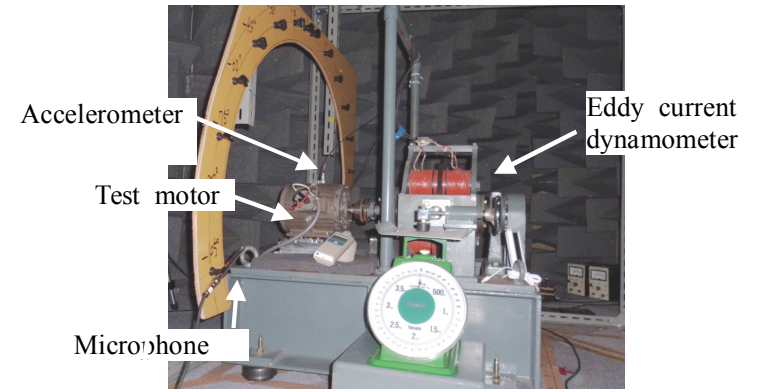

Fig. 2. Experimental apparatus

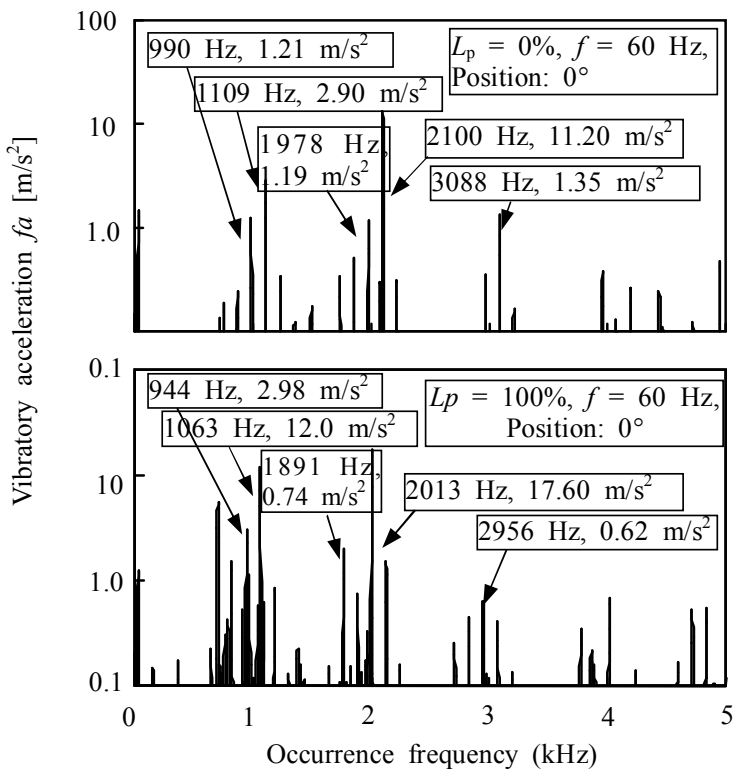

(a) Electromagnetic vibration

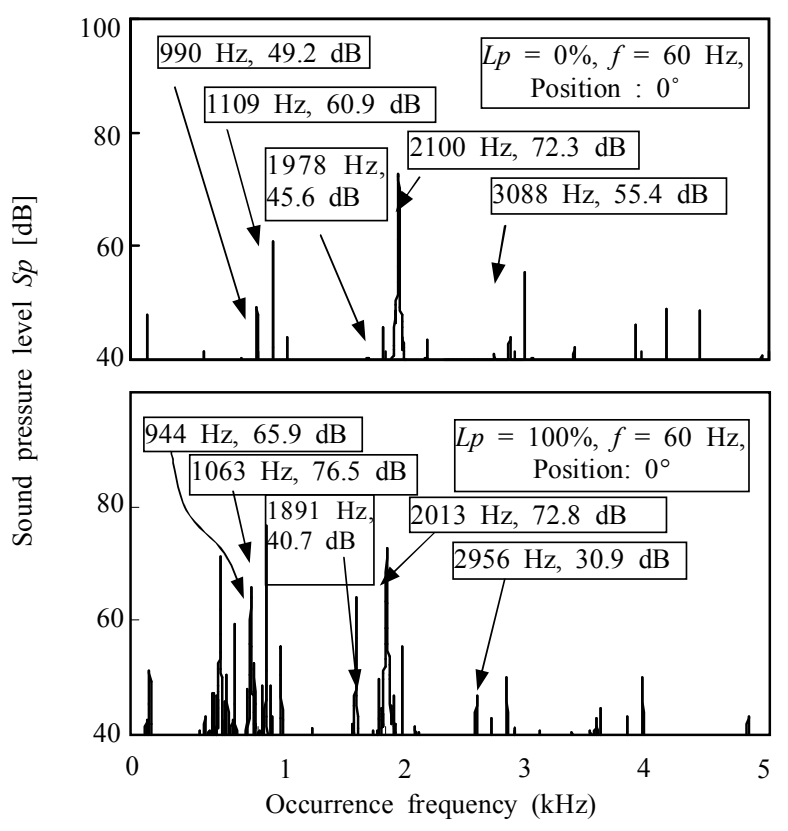

(b) Electromagnetic noise

Fig. 3. Dominant electromagnetic vibration and noise at $0^{\circ}$

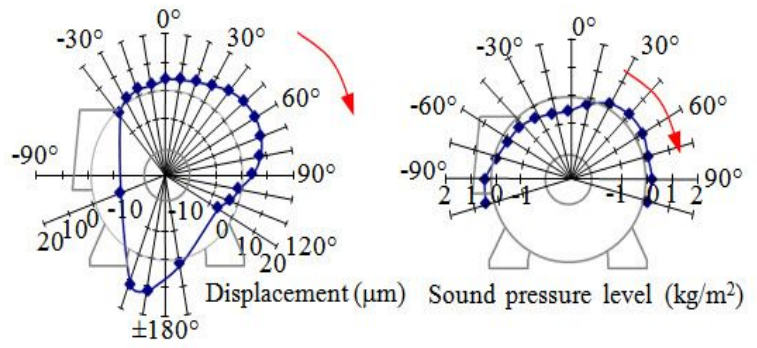

(a) $f=60 \mathrm{~Hz}$

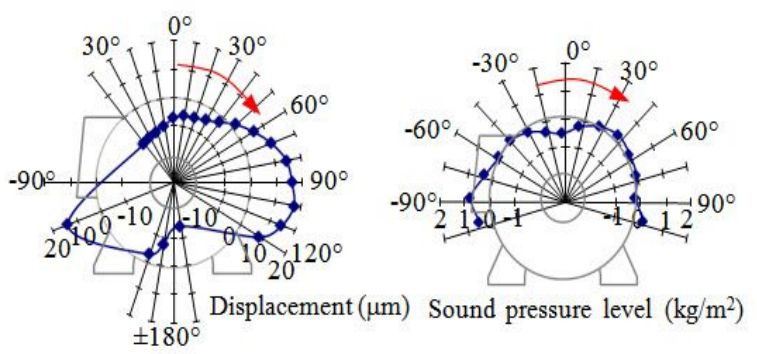

(b) $f_{\mathrm{x}}$ is constant.

$\longrightarrow$ : Direction of rotation of node

Fig. 4. $16.5 f$ component at $L p=100 \%$

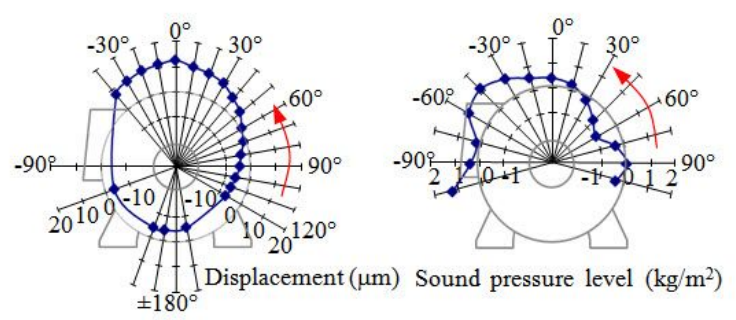

(a) $f=60 \mathrm{~Hz}$ at $L p=0 \%$

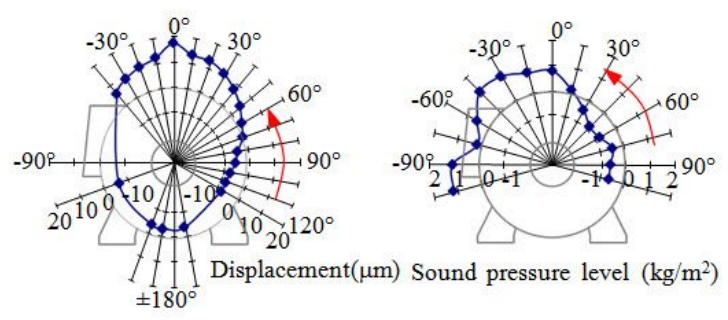

(b) $f=60 \mathrm{~Hz}$ at $L p=100 \%$

$\longrightarrow$ : Direction of rotation of node

Fig. 5. $18.5 f$ component

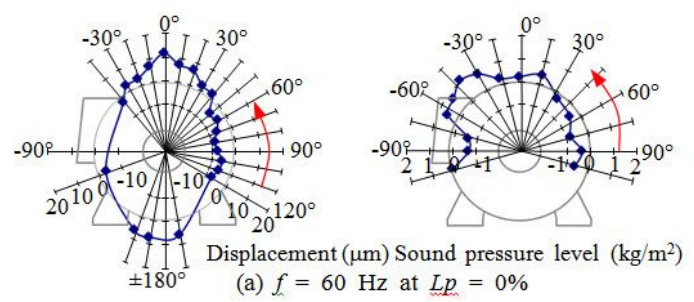

Fig. 6. $33 f$ component (Continued) 


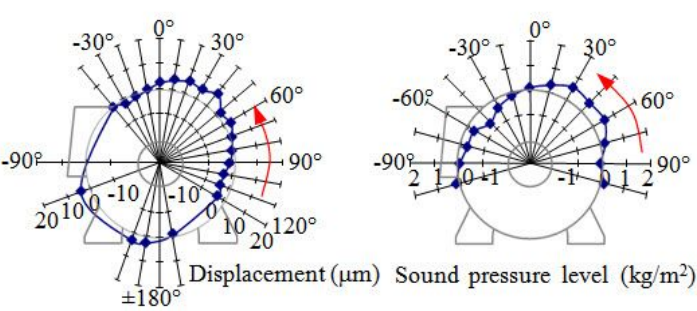

(b) $f=60 \mathrm{~Hz}$ at $\mathrm{Lp}=100 \%$

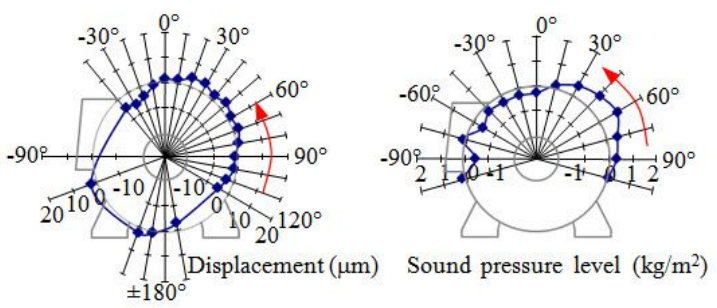

(c) $f_{\mathrm{x}}$ constant at $L p=100 \%$

$\rightarrow$ : Direction of rotation of node

Fig. 6. $33 f$ component

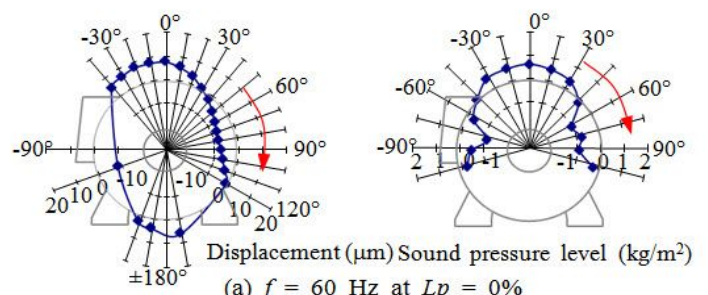

(a) $f=60 \mathrm{~Hz}$ at $L p=0 \%$

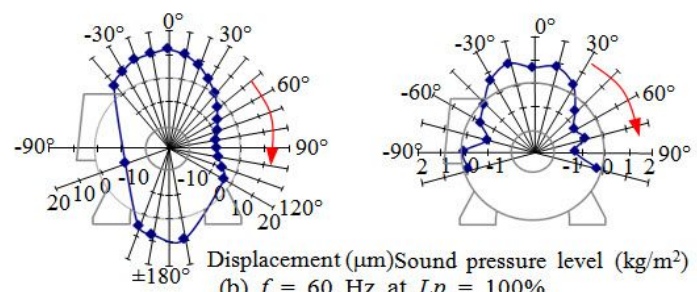

(b) $f=60 \mathrm{~Hz}$ at $\mathrm{Lp}=100 \%$

$\longrightarrow$ : Direction of rotation of node

Fig. 7. $35 f$ component

\section{Acknowledgment}

The authors thank the graduate students of the Department of Electrical Engineering, College of Engineering, Chubu University, for their assistance.

\section{References}

[1] For example, S. J. Yang, "Low-Noise Electrical Motor", Clarendon Press, Oxford, 1981.

[2] K. Tsuboi, I. Hirotsuka, F. Ishibashi, "Causes and Characteristics of the Electromagnetic Vibration of a Squirrel Cage Induction Motor under Load", Trans. IEEJ, Vol. 117D, No. 1, pp. 73-80, January 1997.

[3] Japanese Industrial Standards Committee, JIS C 4210, 2001 (in Japanese).

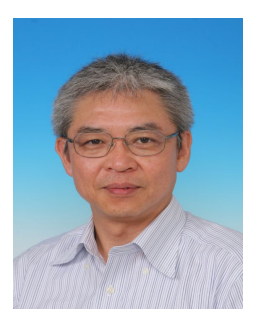

Isao Hirotsuka (Member of I.E.E.J) is a professor at Chubu University, College of Electrical Engineering, Department of Electrical Engineering. He received a Master of Engineering degree in 1986 from Chubu University. $\mathrm{He}$ worked for Mitsubishi Electric Corporation from April to August 1986. He received his Doctor of Engineering degree in 1990 from Chubu University. His major research is on rotating electrical machines, with special emphasis on abnormal phenomena in induction machines. He is a member of IEEE, the Japan Society of Applied Electromagnetics and Mechanics, Institute of Noise Control Engineering of JAPAN, and of the Institute of Electrical Installation Engineers of Japan.

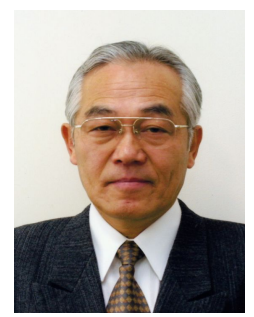

Kazuo Tsuboi (Senior Member of I.E.E.J) is a professor at Chubu University. He received his Master's degree in 1973 from Chubu Institute of Technology (now Chubu University), and his Doctor of Engineering degree in 1976 from Kyushu University. His research interests are in the fields of power, electric drives, and control systems. His major research is on rotating electrical machine. He received a Paper Award from the Institute of Electrical Engineering of Japan (IEEJ). He is a member of IEEE, and of the Institute of Electrical Installation Engineers of Japan.

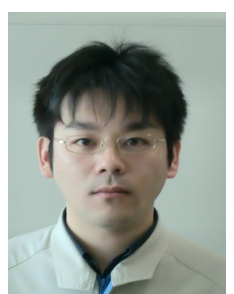

Yousuke Takahashi (Member of I.E.E.J) received a Master of Engineering degree in 2002 from Chubu University. He joined Toshiba industrial products Manufacturing Corporation in 2003. He is mainly engaged in development and design of induction motor. 